Einführung in das Schwerpunktthema

\title{
Forschung \\ für eine Ernährungswende
}

$\mathrm{E}$

Von Ulla Simshäuser Auch im Rahmen der vom Bundesministerium für Bildung und Forschung (BMBF) geförderten sozial-ökologischen Forschung (SÖF) bildet sie zusammen mit Landwirtschaft einen eigenen Förderschwerpunkt. Das hier angesiedelte Verbundforschungsvorhaben Ernährungswende präsentiert sich in diesem Heft erstmals gemeinsam mit Ergebnissen aus seinen Empirien und mit seinem zentralen Forschungsansatz, der KonsumentInnenperspektive (1).

Ernährungswende will dort ansetzen, wo Wissenschaft und Politik bisher aufhörten: am Ernährungsalltag. Sie stellt grundsätzliche Fragen nach der Zukunftsfähigkeit unseres alltäglichen Umgangs mit Ernährung und sie betrachtet diese Grundfrage aus unterschiedlichen Blickwinkeln: der Zukunftsfähigkeit von Ernährungsstilen und ihren Stoffverbräuchen, der Zukunftsfähigkeit von Institutionen und der Zukunftsfähigkeit von Politikmustern und Kommunikationsformen. Die letzten beiden Schwerpunkte werden ergänzt durch europäische Vergleichsstudien. Das Vorhaben wird im Juni 2005 der Öffentlichkeit seine Sozialökologischen Leitlinien einer Ernährungswende vorstellen.

\section{- Die Beiträge im Einzelnen}

Ingrid Keller von der WHO eröffnet den Schwerpunkt mit einer Beschreibung der Herausforderungen, zu denen der Wandel im alltäglichen Ernährungs- und Gesundheitsverhalten für das Gesundheitswesen geführt hat: Sechs von sieben Risikofaktoren für vorzeitigen Tod und schwere chronische Erkrankung sind auf Ernährung und Bewegung zurückzuführen, verbunden mit hohen Gesundheitskosten. Im Mai 2004 wurde deshalb von der WHO eine globale Strategie zur Förderung von Ernährung und Bewegung verabschiedet. Angesichts dieser Dringlichkeit bedarf es zukunftsfähiger alltagstauglicher Strategien für eine Ernährungswende. Die transdisziplinäre Forschung braucht deshalb eine Forschungsperspektive, die den bisher vernachlässigten Alltag von KonsumentInnnen zentral in den Mittelpunkt stellt. Dafür hat das Projekt Ernährungswende die KonsumentInnenperspektive entwickelt. Ulla Simshäuser stellt sie im Schwerpunkt als integrierte Forschungsperspektive erstmals dar. Sie zeigt, wie damit das Handeln von KonsumentInnnen zwischen Markt, Alltag und zivilgesellschaftlicher Orientierung differenziert beschrieben werden kann.

Die folgenden Beiträge veranschaulichen dies. Öffentliche Ernährungskommunikation, so Regine Rehaag, hat in diesem Zusammenhang die Aufgabe, eine öffentliche Verständigung darüber herzustellen, wie wir künftig Ernährung und unseren Ernährungsalltag gestalten wollen und wie Ernährungsverantwortung verteilt werden soll. Zivilgesellschaftliche Beteiligung an diesem Verständigungsprozess ist dafür die Bedingung.

Auf eine weitere Differenzierung weist Doris Hayn hin. Die Ernährungsstile der Deutschen sind so unterschiedlich wie die Alltage von KonsumentInnen. Insgesamt sieben Ernährungsstile werden durch eine repräsentative Umfrage identifiziert und von der Autorin vorgestellt. Diese Lebensstile sind überdies nicht fix, sondern können nach unterschiedlichen Lebensphasen variieren. Insbesondere die so genannten Billig- und Fleischesser haben nach den Befunden ein erhöhtes Gesundheitsrisiko.

Wie aber sehen nachhaltige Ernährungsstile aus? Wie ist der wachsende Trend zum Essen außer Haus in ökologischer Hinsicht zu bewerten? Ulrike Eberle, Uwe Fritsche und Kirstin Wiegmann haben die Stoffströme ausgewählter Ernährungsstile berechnet. Die darauf aufbauende Szenarioanalyse soll Aufschluss über die ökologische Relevanz von unterschiedlichen Lebensstilen für unsere Ernährungszukunft geben. In methodischer Hinsicht ist damit auch der Versuch geglückt, eine Methodenintegration ausgehend von der KonsumentInnenperspektive vorzunehmen.

Über unseren Ernährungsalltag entscheiden wir nicht ausschließlich selbst. In wachsendem Ma- ße leben wir ihn in Institutionen wie Kindergarten, Schule, Betrieb, Krankenhaus. Wir sind auf die dort vorhandene Infrastruktur und die Wahrnehmung einer öffentlichen Ernährungsverantwortung angewiesen. Ulla Simshäuser et al. erläutern am Beispiel der Schulverpflegung, dass diese Ernährungsverantwortung in Deutschland weitgehend fehlt und wie zentral hier - das zeigt der Vergleich mit Schweden - die Bedeutung der Kommunen ist. Die Fokusgruppen des IÖW mit SchülerInnen, LehrerInnen und Eltern zeigen außerdem, dass die Ausgliederung von Ernährung und Gesundheit aus dem Lebensraum Schule weitreichende Folgen für den Erwerb grundlegender Alltagskompetenzen von SchülerInnen hat.

Deshalb muss Schule, so Sigrid Beer in ihrem Beitrag, endlich Ernst machen und das Leben in die Schule holen. Grundlegende Kulturtechniken wie Essen und Ernährung, aber auch Lebensfïhrung und Konsum müssen heute von Schulen vermittelt werden. Die Mitglieder des Projekts Revis haben entsprechende Curricula für eine zeitgemäße Ernährungs- und Verbraucherbildung entwickelt. Die Autorin stellt deren Grundphilosophie in ihrem Beitrag vor.

Veronika Noelle zeigt schließlich, wie eine Ernährungswende praktisch umgesetzt werden kann. Im Projekt OSSENA, das ebenfalls vom BMBF im Rahmen der sozial-ökologischen Forschung gefördert wird, hat sie mit ihrem Team ernährungsbezogene Interventionen in Schulen durchgeführt und in ihrem Beitrag beschrieben. Erste Ergebnisse zeigen: Gesunde Ernährung kann als Teil regionaler Esskultur in der Schule mit hoher Motivation gelernt werden, wenn sie richtig vermittelt wird und möglicherweise auch auf familiale Konsumgewohnheiten rückwirkt.

\section{Anmerkung}

(1) Vgl. auch die Beiträge in: Ernährung im Alltag- wie schmeckt die Zukunft? Öko-Mitteilungen, Informationen aus dem Institut für angewandte Ökologie e.V., Heft №. 4, Freiburg 2004.

\section{Die Autorin}

Dr. Ulla Simshäuser ist wissenschaftliche Mitarbeiterin in den Forschungsfeldern ökologische Produktpolitik und ökologischer Konsum des Instituts für ökologische Wirtschaftsforschung (IÖW).

Kontakt: IÖW Heidelberg, Bergstraße 7,

69120 Heidelberg. Tel. 06221-649160,

E-Mail: Ulla.Simshaeuser@heidelberg.ioew.de 
(c) 20I0 Authors; licensee IÖW and oekom verlag. This is an article distributed under the terms of the Creative Commons Attribution Non-Commercial No Derivates License (http://creativecommons.org/licenses/by-nc-nd/3.o/), which permits unrestricted use, distribution, and reproduction in any medium, provided the original work is properly cited. 